\title{
Neurophysiological studies of autogenous sural nerve grafts
}

\author{
R. T A L L I S, P. ST A N I F O R T H, A N D T . R. F I S H E R \\ From the Departments of Clinical Neurophysiology and Orthopaedics, North Staffordshire Royal \\ Infirmary, Stoke-on-Trent
}

SUM M ARY Sixteen autogenous sural nerve grafts used for ulnar and median nerve injuries in the forearm have been studied neurophysiologically up to two and a half years after operation. Motor and sensory nerve conduction studies revealed a slow but sustained improvement during the follow-up period. By two years, motor conduction velocity across the graft itself reached in most cases 40 to $85 \%$ of the conduction velocity in the contralateral normal limb. Some reduction of motor conduction velocity was observed in the uninjured nerve proximal to the graft but this was less marked. Sensory nerve action potentials were obtained in $44 \%$ of nerves after 18 months although in all cases the amplitude of the potentials and in most cases their velocity, was greatly reduced.

There has been a lack of information about the neurophysiological recovery of human peripheral nerves after repair apart from a single report by Ballantyne and Campbell in 1973 on the results after secondary epineurial repair. We present the results of a similar longitudinal study to assess the neurophysiological changes that occurred during the recovery period of 16 severely injured peripheral nerves of the forearm which were repaired with autogenous sural nerve grafts using the technique described by Millesi et al. in 1972 .

\section{Materials and methods}

Of 25 median and ulnar nerve grafts performed between May 1973 and February 1976, 16 have been followed up (Table 1). Eight grafts, five median and three ulnar, were lost to follow-up, and one median nerve graft was excluded because of anomalous innervation. All the grafts were in the forearm. Six median and six ulnar grafts were at the level of the wrist, one ulnar graft was just distal to the elbow, and the rest were in the midforearm. All patients had undergone interfascicular surval nerve grafting (Millesi et al., 1972, 1976) for defects of $30-150 \mathrm{~mm}$. The interval betweeen injury and grafting varied from one to 19 months but in 12 patients the interval was less than

Address for reprint requests: Dr R. Tallis, Wessex Neurological Centre, Southampton General Hospital, Tremona Road, Southampton SO9 4XY.

Accepted 16 February 1978
Table 1 Details of grafted nerves studied and whether an evoked motor response was obtained

\begin{tabular}{cclll}
\hline Nerve & $\begin{array}{l}\text { Length of } \\
\text { graft } \\
(\mathrm{mm})\end{array}$ & $\begin{array}{l}\text { Follow-up } \\
\text { (months after } \\
\text { operation })\end{array}$ & $\begin{array}{l}\text { Number of } \\
\text { studies }\end{array}$ & $\begin{array}{l}\text { Evoked } \\
\text { motor } \\
\text { response }\end{array}$ \\
\hline Ulnar & & & & \\
1 & 30 & 18 & 10 & Present \\
2 & 40 & 19 & 2 & Present \\
3 & 40 & 42 & 3 & Present \\
4 & 50 & 20 & 2 & Present \\
5 & 50 & 27 & 2 & Present \\
6 & 50 & 27 & 3 & Present \\
7 & 60 & 30 & 4 & Present \\
8 & 70 & 17 & 1 & Absent \\
9 & 70 & 19 & 5 & Present \\
10 & 70 & 24 & 3 & Present \\
Median & & & 2 & Present \\
1 & 40 & 23 & 2 & Present \\
2 & 40 & 24 & 1 & Present \\
3 & 50 & 19 & 3 & Present \\
4 & 50 & 29 & 2 & Absent \\
5 & 90 & 24 & 10 & \\
6 & 150 & 16 & &
\end{tabular}

four months. Fourteen of the nerves were studied on more than one occasion, and a total of 55 studies was performed. The age range of the patients at the time of grafting was from 10-55 years with a mean age of 25 years.

\section{ELECTROMYOGRAPHY}

A DISA type 14 electromyograph was used. Concentric needle electrodes were inserted into the first dorsal interosseous and hypothenar muscles in the case of ulnar nerves, and into abductor pollicis brevis in the case of median nerves. Spon- 
taneous activity (fibrillation and positive sharp waves) was noted if present, as was evidence of motor unit recruitment on voluntary effort. Finally, the electromyographic pattern on maximum voluntary effort was observed and was classified as either reduced or a full interference pattern.

\section{MOTOR CONDUCTION VELOCITIES}

Standard techniques were used for measuring the fastest motor conduction velocity in the median and ulnar nerves, recording the evoked motor response in abductor pollicis brevis or first dorsal interosseous and hypothenar muscles respectively. In each case velocities were measured over the grafted segment, the site of the graft being inferred from the position of the operation scar. In addition, velocities were measured over the segment between the proximal end of the scar and the elbow, this consisting of nerve uninjured at the time of the accident or surgery.

In order to identify anastomoses between the median and ulnar nerves in the forearm, both nerves were stimulated when recording in both ulnar and median supplied muscles. Only one case of the Martin-Gruber anastomosis (Iyer and Fenichel, 1976) was found, and that patient was excluded from the series.

\section{SENSORY STUDIES}

Sensory nerve action potentials (SNAP) were studied by stimulating the digital branches of the relevant nerves, using surface ring electrodes on the appropriate finger (Dawson, 1956). When testing median nerves, the index finger was used and in the case of the ulnar nerve, the little finger. Evoked responses were recorded at the wrist by means of $12 \mathrm{~mm}$ tin plate DISA $13 \mathrm{~K}$ electrodes. The recording area was prepared before application of the electrodes by abrasion with sandpaper to reduce skin impedance. The electrodes were coated with electrode jelly. A rectangular electrical impulse $0.2 \mathrm{~ms}$ in duration was delivered at the maximum comfortable voltage. The recording electrodes at the wrist were placed at the site at which an evoked motor response had had the lowest thresholds to stimulation. The small SNAPs recorded were distinguished from background noise using a digital averager (DISA type 14), and the averaged response to 256 stimuli was recorded and stored on the oscilloscope. The peak-to-peak amplitude of the response was measured by comparing it with the calibration signal generated simultaneously by the averager.

Motor and sensory studies were performed on the corresponding nerve of the contralateral nor- mal limb so that each parameter (latency, velocity, amplitude, evoked motor response, and SNAP) could be compared with control values obtained from the same subject. In the analysis of results each parameter has been expressed as a percentage of that obtained on the normal side, which, therefore, serves as an internal control. Absolute values were not considered useful in view of the wide variation between individuals in normal nerves and even within individuals on different occasions. Temperatures were recorded at all sites by means of a DISA type 15 G05 temperature unit. The skin temperature during examination lay between 34 and $36^{\circ} \mathrm{C}$.

\section{Results}

\section{ELECTROMYOGRAPHY}

In all tests performed during the early postoperative period the muscles exhibited profuse fibrillation and positive sharp waves and an absence of recruitment on voluntary effort. Recruitment of motor units was not observed before five months after grafting but its appearance usually coincided with the first appearance of an evoked motor response on stimulating the nerve. When studying ulnar nerves, recruitment was always noted earlier in hypothenar muscles than in the first dorsal interosseous muscle. By the end of the second postoperative year recruitment had been observed in muscles innervated by 14 out of the 16 grafts, and a full interference pattern was seen in seven of these. In four of the reinnervated hands positive sharp waves and fibrillation potentials were observed to persist even at this late stage.

\section{MOTOR STUDIES}

Evoked motor responses were recorded in 14 of the 16 nerves, providing unequivocal evidence of the establishment of neurophysiological continuity after nerve grafting (Table 1). Distal and elbow latencies showed a progressive reduction with time postoperatively, consistent with overall improved conduction in the neuromuscular unit. Even after 30 months, however, the distal latencies remained abnormally prolonged (Fig. 1).

As expected, conduction velocity over the grafted segment was much reduced postoperatively, lying mostly between 20 and $50 \%$ of normal by the end of the first year, and mostly between 40 and $85 \%$ of normal by the end of the second year (Fig. 2). The conduction velocity was also reduced in the uninjured segment of nerve situated proximal to the graft below the elbow (Fig. 3). A gradual improvement with time postoperatively was apparent in both these segments during the 


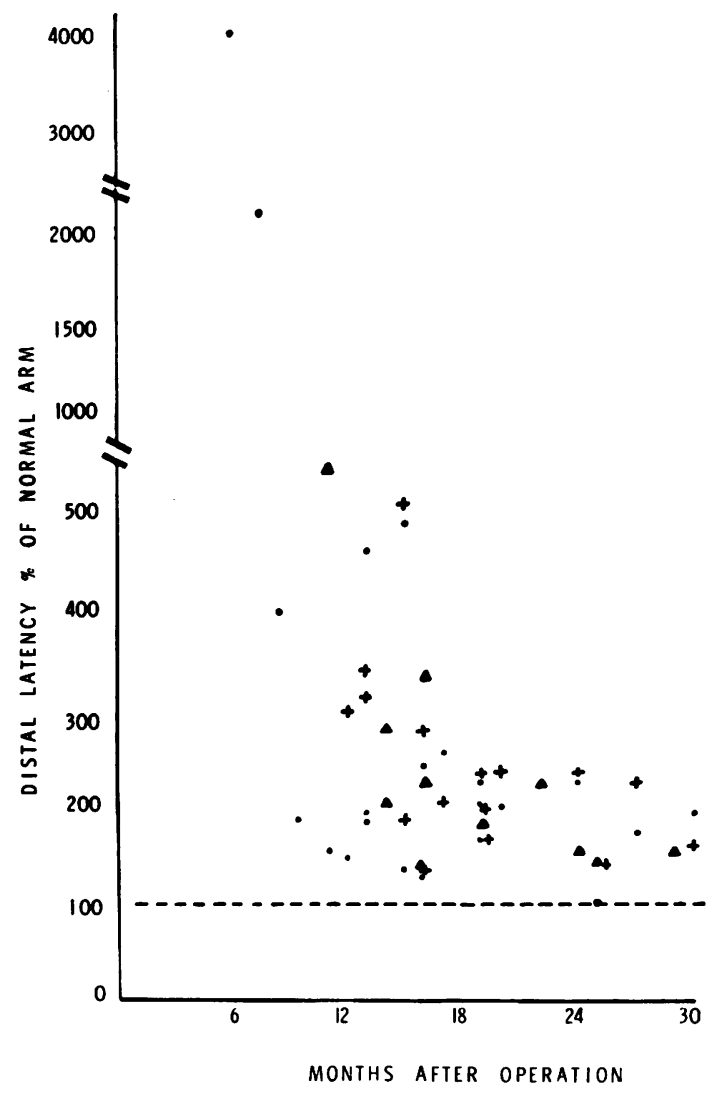

Fig. 1 Distal motor latencies as a percentage of control values; $\bullet=$ hypothenar muscles-ulnar, $+=$ first dorsal interosseous-ulnar; $\Delta=$ thenar muscles-median. period of study (Figs. 2 and 3). When studying the ulnar nerve grafts, conduction velocities were measured over these segments to both the hypothenar muscles and to first dorsal interosseous muscle.

The conduction velocities have been plotted separately in Figs. 2 and 3, and it can be seen that the conduction velocity over the proximal segment of nerve and the grafted segment was generally faster to the hypothenar muscles than to the first dorsal interosseous muscle, even though the identical segment was studied on the same occasion. In one patient studied at 18 months, and two patients studied at 24 months, abnormally fast conduction velocities were recorded proximal to the nerve grafts. This could have resulted from technical difficulties when stimulating the nerve at the proximal end of the nerve graft and to the short distances involved.

\section{SENSORY STUDIES}

Sensory nerve action potentials (Table 2) were recorded on 11 occasions in seven nerves, clearly indicating sensory neurophysiological recovery after sural nerve grafting. With the exception of case 1, SNAPs were not recorded within the first 18 months after operation. The conduction velocity and the amplitude of the SNAPs, expressed as a percentage of the normal side, were usually markedly reduced.

CLINICAL RESULTS

Recovery of muscle power was assessed using the Medical Research Council grading (Medical Research Council, 1954) and correlated only approxi-

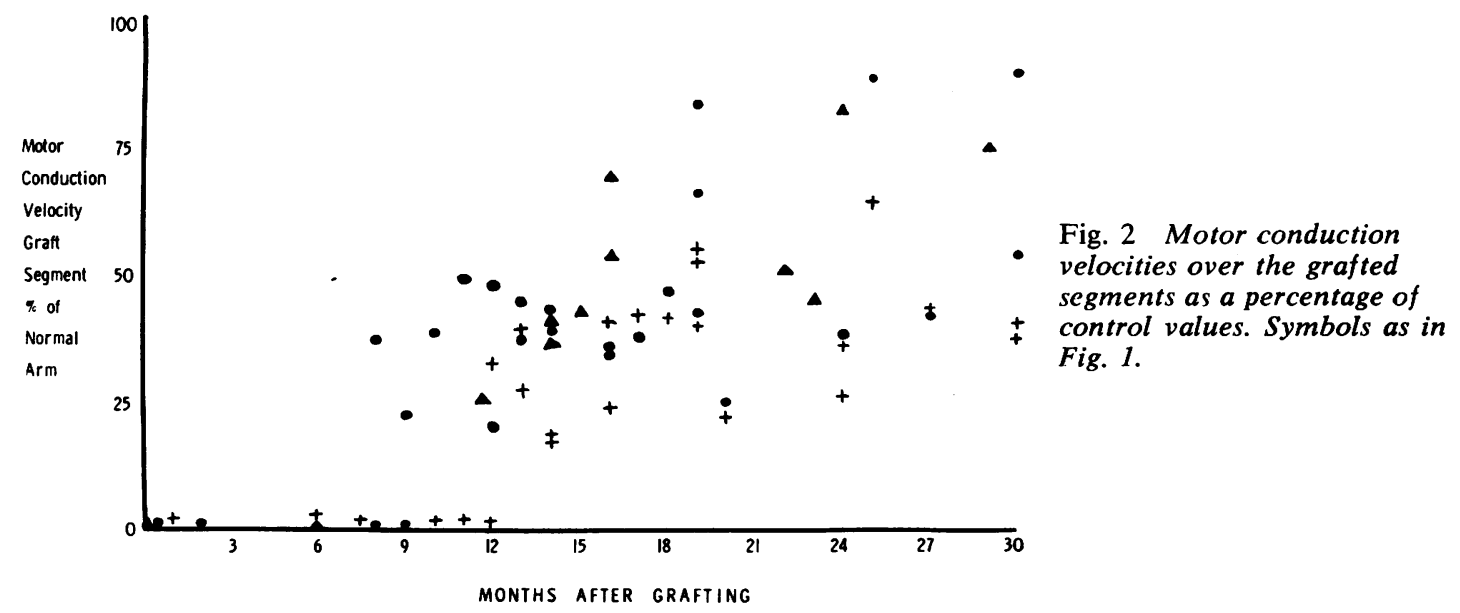




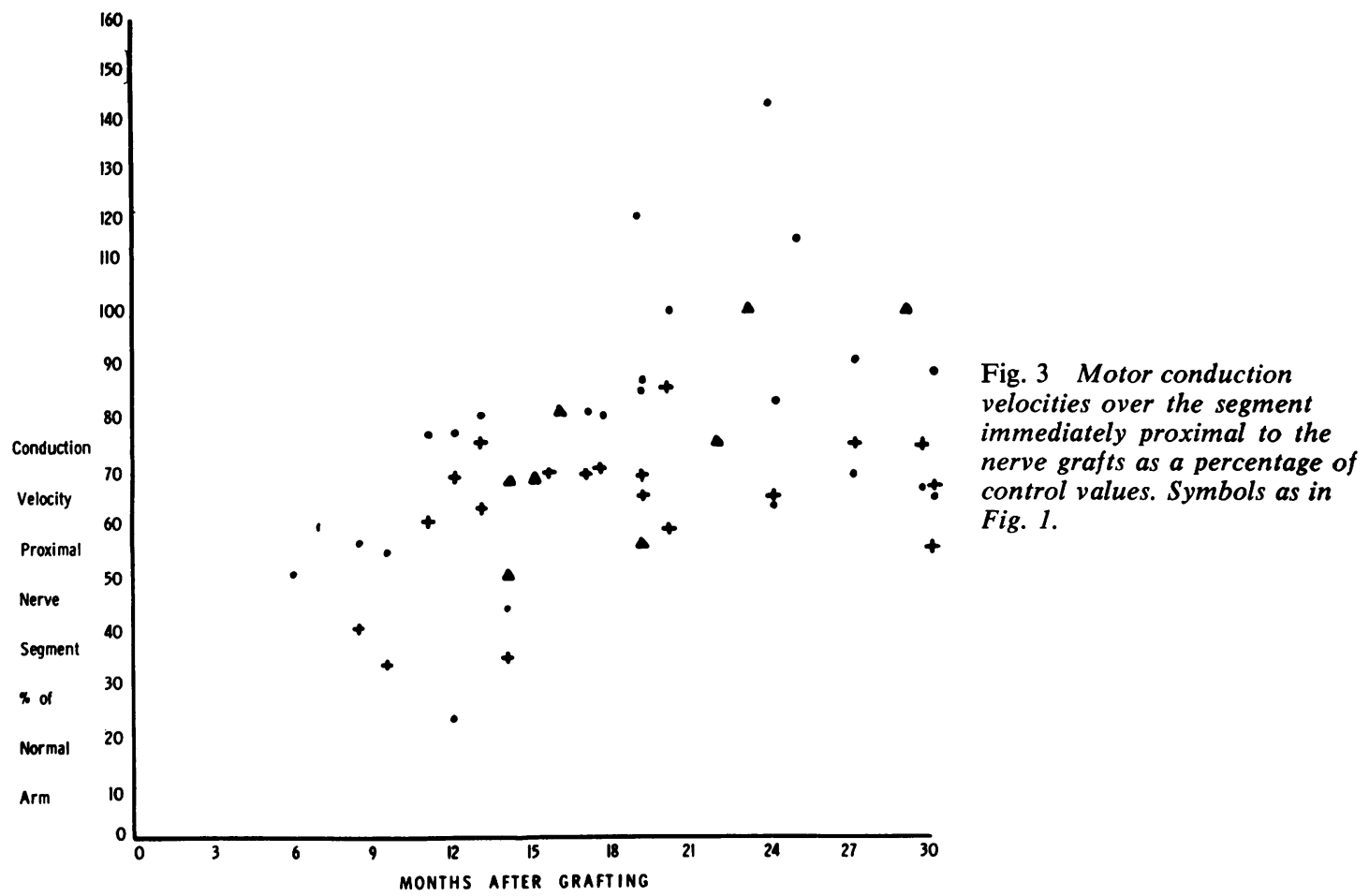

mately with the neurophysiological findings (Fig. 4). Furthermore, this correlation applies only to the group as a whole and is not close enough for predictions to be made in an individual case concerning the degree of recovery on the basis of the neurophysiological findings at a given time after operation. Where sensory recovery was judged to be S3 or better, SNAPs were always obtained. Where sensory recovery was less than S2 they were never obtained.

\section{Discussion}

Sural nerve grafting was introduced by Millesi for repair of nerve defects as an alternative to secondary epineurial suture which is said to be unsatisfactory because of the tension produced at the suture line. The harmful effects of tension at the suture line have been well documented experimentally (Highet and Sanders, 1943) and clinically (Nicholson and Seddon, 1957).

Table 2 Clinical recovery, sensory conduction velocities, and sensory nerve action amplitudes in those grafted nerves from which sensory evoked potentials could be obtained

\begin{tabular}{|c|c|c|c|c|c|c|c|}
\hline & & & \multirow[b]{2}{*}{$\begin{array}{l}\text { Clinical recovery } \\
\text { (MRC grades) }\end{array}$} & \multicolumn{3}{|c|}{ Sensory nerve action potentials } & \multirow[b]{2}{*}{ Potentials amplitude $(\mu V)$} \\
\hline \multicolumn{3}{|c|}{ Nerve } & & $\begin{array}{l}\text { Follow-up } \\
\text { (months after } \\
\text { operation) }\end{array}$ & $\begin{array}{l}\text { Velocity } \\
\text { (\% \%ormal } \\
\text { side) }\end{array}$ & $\begin{array}{l}\text { Amplitude } \\
\text { (\% normal } \\
\text { side) }\end{array}$ & \\
\hline 1 & Ulnar & (a) & S2 & 13 & 11.5 & 24 & 3 \\
\hline & & (b) & S2 & 15 & 10 & 27 & 4 \\
\hline & & (c) & S2 & 17 & 17 & 38 & 5 \\
\hline 2 & Ulnar & & $\mathbf{S} 2+$ & 19 & 15 & 12 & 3 \\
\hline 3 & Ulnar & (a) & $\mathbf{S 2}+$ & 24 & 68 & 10 & 2.5 \\
\hline & & (b) & S3 & 27 & 66 & 10 & 2 \\
\hline 4 & Ulnar & (a) & S2+ & 19 & 19 & 7 & 2 \\
\hline & & (b) & & 30 & 50 & 9 & 2.5 \\
\hline 5 & Ulnar & & s3+ & 42 & 7 & 46 & 4.6 \\
\hline 6 & Median & & s3+ & 24 & 58 & 14 & 2 \\
\hline 7 & Median & & S3+ & 29 & 25 & 23 & 6 \\
\hline
\end{tabular}




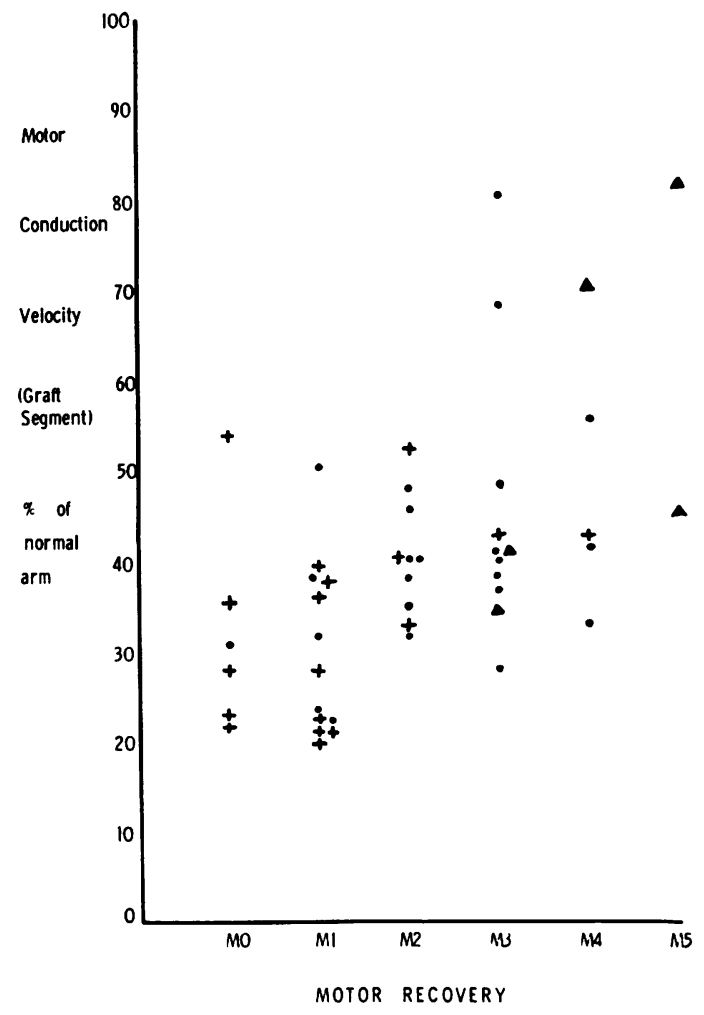

Fig. 4 Motor conduction velocities over the grafted segments, given as a percentage of control values, plotted against motor recovery (MRC grades). Symbols as in Fig. 1.

A comparison of different surgical procedures is difficult because of the problem of assessing clinical improvement and of distinguishing improvement due to peripheral regeneration from that related to adaptive central nervous system changes, as in the development of trick movements and the process of sensory re-education. Electromyographic and nerve conduction studies enable peripheral changes to be distinguished and isolated from adaptive changes in the central nervous system. With the exception of the recent abstract (Yakubovich, 1977), we are unaware of any previous reports on the electrophysiological status of patients after nerve grafting.

In all cases the muscles supplied by the injured nerves initially showed the characteristic electromyographic features of denervation. The appearance of recruitment on voluntary effort and the observation of an evoked motor response on stimulating the nerve showed that some axons had regenerated.
Evoked motor responses were not obtained in the first five months after operation though the exact timing of their appearance in individual cases was not ascertained because of the impracticability of examining patients repeatedly at frequent intervals. An evoked motor response was obtained first on stimulating proximally to the graft. Since the measurement of motor conduction velocity depends on obtaining an evoked motor response on stimulating the nerve at two different sites, motor conduction velocity could not be measured in the early stages. Once measurable, however, the motor conduction velocity showed a progressive increase across the grafted segment of the injured nerve up to two years after operation. It appears from the few cases we studied beyond the second year that limited change occurs in the third year. The fastest conduction velocities measured at this time mostly range from 40 to $85 \%$ of the normal side. Yakubovich also noted persistently low conduction velocities. After epineurial repair in humans, motor conduction velocities distal to the site of repair reached a plateau after 10 months when they were within the normal range (Ballantyne and Campbell, 1973).

Animal studies have shown that, after transection of a nerve, the newly regenerated axons have a very small diameter and a very thin myelin sheath, both of these factors leading to a very slow rate of conduction. Over the succeeding 1224 months, both axon diameter and myelin sheath thickness reach almost normal values, and the maximum conduction velocities rise to $60-95 \%$ of normal (Berry et al., 1944; Sanders and Whitteridge, 1946). The internodal length, however, remains greatly reduced indicating that this is not important in conduction velocity (Cragg and Thomas, 1957). Studies on grafted sciatic nerve in dogs (Schroder, 1972) showed that neither the average maximum fibre diameter nor the thickness of the myelin sheath increased significantly beyond 12-24 months after operation. By this time the diameter of regenerating axons had reached about $80 \%$ of normal and the myelin sheath was only fifty per cent of normal thickness. On the basis of this animal study, a persistently reduced motor conduction velocity in the grafted nerves studied here might be expected.

The reduction of conduction velocity in the segment immediately proximal to the grafted segment was interesting. Several authors (Cragg and Thomas, 1961; Aitken and Thomas, 1962) have described degeneration proximal to nerve lesions. Such changes might be partially due to distal flow of axoplasm. It has also been observed that the amount of ascending regeneration may be 
greater after trauma than after a clean surgical section (Seddon, 1975). The present findings have shown that the conduction velocity in the proximal segment has remained below the normal value, though it is not possible to say how far proximal to the lesion this neurophysiological abnormality extended.

The distal latency was prolonged as late as 30 months after operation. This might be due in part to persistent defects of neuromuscular transmission as was discussed by Ballantyne and Campbell (1973).

Sensory nerve action potentials could be recorded 18 months after operation. This was always associated with some clinical recovery. The long delay in the appearance of recordable SNAPs was a reflection of their dependence upon recovery of function in the most distal part of the nerve, namely that segment from the fingertip to the wrist. In addition, the height of the SNAP would depend on the number and diameter of functioning sensory fibres (Terzis et al., 1976) as well as on the synchronisation of the time of arrival of the impulses at the site of the recording electrodes. In all cases there was a very marked reduction of SNAP amplitude and conduction velocity as compared with that obtained on the normal side. Yakubovich (1977) was, with a single exception, unable to record SNAPs in 16 grafts.

Correlation between neurophysiological findings and motor and sensory recovery were not sufficiently close to allow predictions regarding the degree of recovery in an individual patient at any particular time after operation.

The finding that the same segment of ulnar nerve had a faster conduction velocity when the evoked response was recorded in the hypothenar muscles than when it was recorded in the first interosseous muscle semed to indicate the earlier regeneration of fibres to the more proximal muscle than to the more distal one. This was consistent with the observation that recruitment and evoked motor response could be detected earlier in the hypothenar muscles than in the first dorsal interosseous muscle.

These studies clearly illustrated that neurophysiological continuity was achieved in grafted nerves in nearly all cases and that progressive regeneration affecting both motor and sensory fibres continued for at least two years after operation. They provided basic information on peripheral regeneration after this type of repair, and indicated a continuing peripheral contribution to recovery up to at least two years after grafting.
We would like to thank Dr E. C. Hutchinson and Dr G. Boddie for allowing us the use of the facilities of the Department of Neurology at the North Staffordshire Royal Infirmary, and Dr E. M. Sedgwick of Wessex Neurological Centre for advice in preparation of this paper.

\section{References}

Aitken, J. T., and Thomas, P. K. (1962). Retrograde changes in fibre size following nerve section. Journal of Anatomy, 96, 121.

Ballantyne, J. P., and Campbell, M. J. (1973). Electrophysiological study after a surgical repair of sectioned human peripheral nerves. Journal of Neurology, Neurosurgery, and Psychiatry, 36, 797-805.

Berry, C. M., Grundfest, H., and Hinsey, J. C. (1944). The electrical activity of regenerating nerves in the cat. Journal of Neurophysiology, 7, 103-115.

Cragg, B. G., and Thomas, P. K. (1957). The relationship between conduction velocity and diameter and internodal length of peripheral nerve fibres. Journal of Physiology (London), 136, 606-614.

Cragg, B. G., and Thomas, P. K. (1961). Changes in conduction velocity and fibres proximal to peripheral nerve lesions. Journal of Physiology (London), 157, 315.

Dawson, G. D. (1956). The relative excitability and conduction velocity of sensory and motor nerve fibres in man. Journal of Physiology (London), 131, 436-451.

Highet, W. D., and Sanders, F. K. (1943). The effect of stretching nerves after suture. British Journal of Surgery, 30, 355.

Iyer, V., and Fenichel, G. M. (1976). Normal median nerve proximal latency in carpal tunnel syndromea clue to coexisting Martin-Gruber anastomosis. Journal of Neurology, Neurosurgery, and Psychiatry, 39, 449.

Medical Research Council (1954). Peripheral Nerve Injuries. Special Report Series, no. 282. Her Majesty's Stationery Office: London.

Millesi, H., Meissl, G., and Berger, A. (1972). The interfascicular grafting of median and ulnar nerves. Journal of Bone and Joint Surgery, 54A, 727.

Millesi, H., Meissl, G., and Berger, A. (1976). Further experience with interfascicular grafting of the median, ulnar and radial nerves. Journal of Bone and Joint Surgery, 58A, 209-218.

Nicholson, O. R., and Seddon, H. J. (1957). Nerve repair in civil practice: results of treatment of median and ulnar nerve lesions. British Medical Journal, 2, 1065.

Sanders, F. H., and Whitteridge, D. (1946). Conduction velocity and myelin thickness in regenerating nerve fibres. Journal of Physiology (London), 105, $152-174$.

Schroder, J. M. (1972). Altered ratio between axon diameter and myelin sheath thickness in regenerated nerve fibres. Brain Research, 45, 49-65. 
Seddon, H. J. (1975). Surgical Disorders of Peripheral Nerves. Second edition. Churchill Livingstone: Edinburgh and London.

Terzis, J. K., Dykes, M. D., and Hakstian, R. W. (1976). Electrophysiological recordings in peripheral nerve surgery. Journal of Hand Surgery, 1, 56.

Yakubovich, E. (1977). Electromyographic follow-up study of fascicular nerve grafts in the upper extremity. Journal of Hand Surgery, 2, 162. 